UOT $547.97+535.37$

\title{
THE INVESTIGATION OF REACTION OF VARIOUS THIOPHENE BASED KNOEVENAGEL ADDUCTS WITH ACETOACETANILIDE
}

\author{
F.N. Naghiyev, A.M. Maharramov, A.R. Asgarova, A.G. Rahimova, \\ M.A. Akhundova, I.G. Mamedov \\ Baku State University \\ 23, Z.Khalilov str., AZ-1148 Baku, Azerbaijan; e-mail: farid.orgchemist@gmail.com
}

Received 15.05.2018

\begin{abstract}
The reaction of Michael addition interaction of 3-phenyl-2-(thiophene-2-carbonyl)acrylonitrile, 2-(thiophene-2-carbonyl)-3-(p-tolyl)acrylonitrile, 3-(4-methoxyphenyl)-2-(thiophene-2-carbonyl)acrylonitril, as well as 3-pyridinyl-2-(thiophene-2-carbonyl)acrylonitrile with acetoacetanilide made it possible to produce substituted hexanones and 3,4-dihydro-2H-pyrane derivatives. Structures of synthesized compounds were acknowledged by NMR and $X$-Ray structural analysis.
\end{abstract}

Keywords: thiophene, acrylonitrile, acetoacetanilide, pyran, NMR

\section{INTRODUCTION}

Pyranes and cyclic ketones are biologically active compounds to make up a structural part of natural compounds [1-2]. New [4+2] annulations were carried out in the presence of triphenylphosphine catalyst and high yield synthesized functionalized dihydropyranes [3]. A similar reaction of $\alpha$ valin derived phosphine was used as catalyst in another work [4]. Also, new cyclation was carried out for $\alpha, \beta$-unsaturated ketones through the use of DABCO-catalyst and synthesized diastereomeric dihydropyrane derivatives [5]. A cascade reaction of malononitrile and $\alpha$ substituted calcone allowed to synthesize chiral multi-substituted amino-4H-pyrane derivatives. Alkoloids were checked up as catalyst in pyrane synthesis reactions [6].

The reaction of isatilidenmalononitriles with malononitriles and 2-tiophenmethylamine (or furfuril amine) one-pot three component condensation was carried out for the first time to obtain appropriate spiropiridines [7].

\section{RESULTS AND DISCUSSION}

We carried out an experiment through the use of the Michael addition reaction for 2(thiophene-2-carbonyl)-3-(p-tolyl)acrylonitrile and 3-(4-methoxyphenyl)-2-(thiophene-2-carbonyl)acrylonitrile with acetoacetanilide at room temperature in methanol media using of 23 drops of methylpiperazine to obtain corresponding hexanone-substituted derivatives. As follows from the reaction mechanism, at the first stage the Michael addition of methylene active compound to double bond takes place and from the obtained Michael adduct (3) forms $\mathrm{CH}_{2}$-anion by action of base. At the last stage this anion atacks a carbonyl group of anion to synthesize an appropriate reaction products(5). 

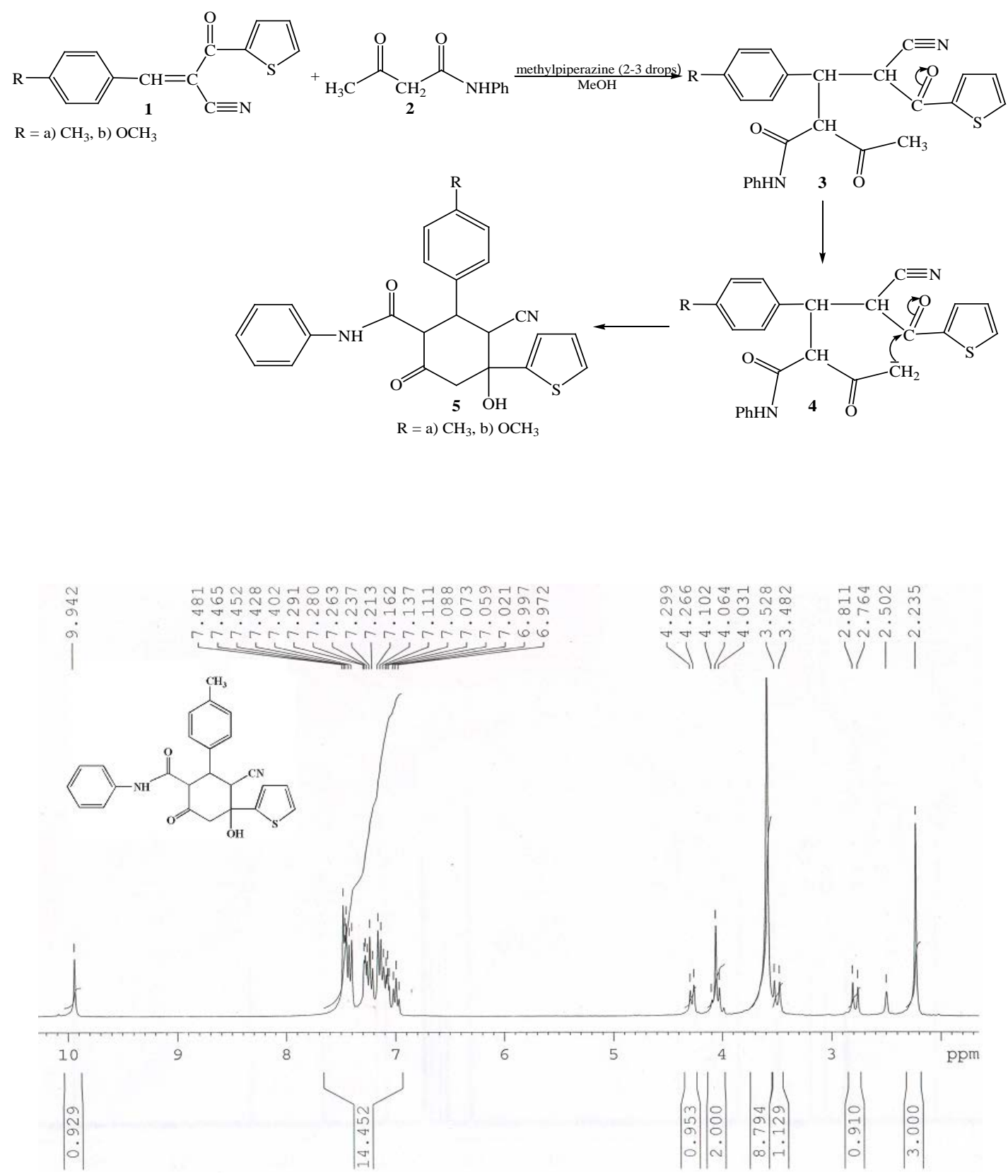

${ }^{1} \mathrm{H}$ NMR spectra of 3-cyano-4-hydroxy-6-oxo-N-phenyl-4-(thiophen-2-yl)-2-(p-tolyl)cyclohexane-1-carboxamide (5a)

Subsequently by means of the Michael addition reaction of Knoevenagel adduct obtained as a result of interaction between pyridine aldehyde and 2-thenoylacetonitrile, with acetoacetanilide in ethanol-water media through the use of 2-3 drops of methylpiperazine to obtain appropriate 3,4dihydro- $2 H$-pyrane derivatives. 

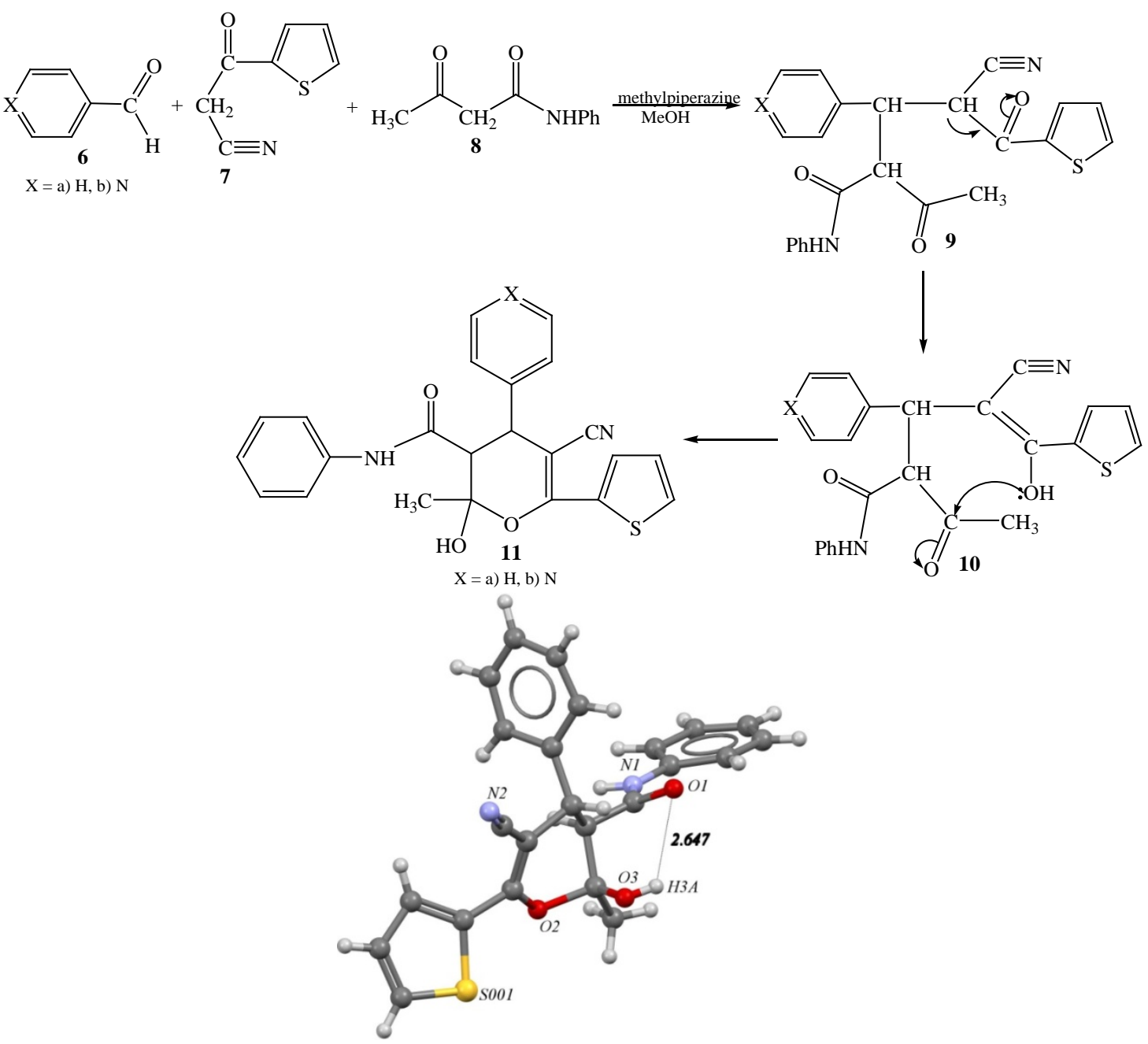

X-Ray structure of 5-cyano-2-hydroxy-2-methyl-N,4-diphenyl-6-(thiophen-2-yl)-3,4-dihydro-2Hpyran-3-carboxamide (11a)

\section{EXPERIMENTAL PART}

It should be noted that all used reagents were bought from Merc and Fluca and used without cleaning. The melting points of compounds were measured at STUART SPM30. Purity of synthesized compounds were checked by TLC, and structures acknowledged on "Bruker 300" NMR apparatus (300 and $75 \mathrm{MHz}$ ).

3-Cyano-4-hydroxy-6-oxo-N-phenyl4-(thiophen-2-yl)-2-(p-tolyl)cyclohexane-1carboxamide (5a): Mixture of 2-(thiophene-2carbonyl)-3-(p-tolyl)acrylonitrile $(2.9 \mathrm{mmol})$ and acetoacetanilide ( $3 \mathrm{mmol}$ ) dissolved in 35 $\mathrm{ml}$ of methanol was stirrered for 5-7 minutes and a 2-3 drop methylpiperazine was added, so the stirring was continued. The reaction progress was tracked by TLC (EtOAc/nhexane, 2:1) and the mixture kept quietly for
24-48 hours. Through evaporating of solvent it became possible to precipitate crystals. Note that crystals were separated by filter paper and recrystalliized from ethanol (95\%) - water mixture, yield $0.89 \mathrm{~g}, 71.60 \%$. mp. $210^{\circ} \mathrm{C}$.

${ }^{1} \mathrm{H}$ NMR (300 MHz, DMSO-d6), $\delta$, m.h.: 2.23 (s, 3H, $\left.\mathrm{CH}_{3}\right) ; 2.79\left(\mathrm{~d}, 1 \mathrm{H}, \mathrm{CH},{ }^{3} \mathrm{~J}_{\mathrm{H}-\mathrm{H}}\right.$ $=14.1) ; 3.50\left(\mathrm{~d}, 1 \mathrm{H}, \mathrm{CH},{ }^{3} \mathrm{~J}_{\mathrm{H}-\mathrm{H}}=13.8\right) ; 4.06$ (s, $\left.2 \mathrm{H}, \mathrm{CH}_{2}\right) ; 4.28$ (d, $1 \mathrm{H}, \mathrm{CH},{ }^{3} J_{\mathrm{H}-\mathrm{H}}=9.9$ ); 6.97-7.48 (m, $\left.12 \mathrm{H}, 9 \mathrm{Ar}-\mathrm{H}+3 \mathrm{CH}_{\text {thiophenyl }}\right) ; 9.94$ (s, $1 \mathrm{H}, \mathrm{NH}) .{ }^{13} \mathrm{C}$ NMR spektr (DMSO-d6), $\delta$, m.h.: $21.14\left(\mathrm{CH}_{3}-\mathrm{Ar}\right), 44.26(\mathrm{CH}), 47.40(\mathrm{CH})$, $54.07\left(\mathrm{CH}_{2}\right), 62.64(\mathrm{CH}), 75.29$ (C $\left.\mathrm{C}_{\text {quat. }}\right), 119.02$ (CN), $119.49\left(2 \mathrm{CH}_{\text {arom }}\right), 123.87\left(\mathrm{CH}_{\text {thiophenyl }}\right)$, $124.45\left(\mathrm{CH}_{\text {arom }}\right), 125.71\left(\mathrm{CH}_{\text {thiophenyl }}\right), 127.63$ $\left(\mathrm{CH}_{\text {thiophenyl }}\right), \quad 128.75 \quad\left(2 \mathrm{CH}_{\text {arom }}\right), \quad 129.14$ $\left(2 \mathrm{CH}_{\text {arom }}\right), 129.54 \quad\left(2 \mathrm{CH}_{\text {arom }}\right), \quad 137.06 \quad\left(\mathrm{C}_{\mathrm{ar}}\right)$, 
$137.17\left(\mathrm{C}_{\mathrm{ar}}\right), 139.14\left(\mathrm{C}_{\mathrm{ar}}\right), 150.57$ ( $\left.\mathrm{C}_{\text {thioph }}\right)$, 165.85 ( $\mathrm{CONH}), 203.12(\mathrm{C}=\mathrm{O})$.

Found, \%: $69.70 \quad \mathrm{C} ; 5.07 \quad \mathrm{H}$ $\mathrm{C}_{25} \mathrm{H}_{22} \mathrm{~N}_{2} \mathrm{O}_{3} \mathrm{~S}$. Calculated, \%: $69.77 \mathrm{C} ; 5.12 \mathrm{H}$.

3-Cyano-4-hydroxy-2-(4-methoxy- phenyl)6-oxo-N-phenyl-4-(thiophen-2-yl)- cyclohexane-1-carboxamide (5b): it was synthesized in the same condition (yield $1.1 \mathrm{~g}, 85.61 \%$ ). $\mathrm{mp} .208^{\circ} \mathrm{C}$.

${ }^{1} \mathrm{H}$ NMR (300 MHz, DMSO-d6), $\delta$, m.h.: $2.77\left(\mathrm{~d}, 1 \mathrm{H}, \mathrm{CH},{ }^{3} \mathrm{~J}_{\mathrm{H}-\mathrm{H}}=14.1\right) ; 3.48(\mathrm{~d}$, $\left.1 \mathrm{H}, \mathrm{CH},{ }^{3} J_{\mathrm{H}-\mathrm{H}}=15.3\right)$; $3.69\left(\mathrm{~s}, 3 \mathrm{H}, \mathrm{CH}_{3} \mathrm{O}\right)$; $4.03\left(\mathrm{~s}, 2 \mathrm{H}, \mathrm{CH}_{2}\right) ; 4.27\left(\mathrm{~d}, 1 \mathrm{H}, \mathrm{CH},{ }^{3} \mathrm{~J}_{\mathrm{H}-\mathrm{H}}=\right.$ 9.6); 6.89-7.47 (m, $\left.12 \mathrm{H}, 9 \mathrm{Ar}-\mathrm{H}+3 \mathrm{CH}_{\text {thiophenyl }}\right)$; $9.93(\mathrm{~s}, 1 \mathrm{H}, \mathrm{NH}) .{ }^{13} \mathrm{C}$ NMR spektr (DMSOd6), $\delta$, m.h.: $43.88(\mathrm{CH}), 47.51(\mathrm{CH}), 54.06$ $\left(\mathrm{CH}_{2}\right), \quad 55.37 \quad\left(\mathrm{CH}_{3} \mathrm{O}\right), \quad 62.79(\mathrm{CH}), \quad 75.22$ ( $\left.\mathrm{C}_{\text {quat. }}\right), 114.23\left(2 \mathrm{CH}_{\text {arom }}\right), 119.07$ (CN), 119.52 $\left(2 \mathrm{CH}_{\text {arom }}\right), \quad 123.87 \quad\left(\mathrm{CH}_{\text {thiophenyl }}\right), \quad 124.42$ $\left(\mathrm{CH}_{\text {arom }}\right), \quad 125.70 \quad\left(\mathrm{CH}_{\text {thiophenyl }}\right), \quad 127.62$ $\left(\mathrm{CH}_{\text {thiophenyl }}\right), \quad 129.14 \quad\left(2 \mathrm{CH}_{\text {arom }}\right), \quad 129.98$ (2CHarom), $131.96\left(\mathrm{C}_{\mathrm{ar}}\right), 139.13\left(\mathrm{C}_{\mathrm{ar}}\right), 150.59$ $\left(\mathrm{C}_{\text {thioph}}\right), 158.90\left(\mathrm{O}-\mathrm{C}_{\mathrm{ar}}\right), 165.90(\underline{\mathrm{CONH}})$, $203.14(\mathrm{C}=\mathrm{O})$.

Found, \%: $67.50 \quad \mathrm{C} ; 4.45 \mathrm{H}$. $\mathrm{C}_{25} \mathrm{H}_{20} \mathrm{~N}_{2} \mathrm{O}_{4} \mathrm{~S}$. Calculated, \%: $67.57 \mathrm{C} ; 4.50 \mathrm{H}$. 5-Cyano-2-hydroxy-2-methyl-N,4-diphenyl6-(thiophen-2-yl)-3,4-dihydro-2H-pyran-3carboxamide (11a):

Mixture of 3-phenyl-2-(thiophene-2carbonyl)acrylonitrile $(2.9 \mathrm{mmol})$ and acetoacetanilide ( $3 \mathrm{mmol}$ ) dissolved in $35 \mathrm{ml}$ of methanol was stirrered for 5-7 minutes and a 2-3 drop methylpiperazine was added, so the stirring was continued. Reaction progress was tracked by TLC (EtOAc/n-hexane, 2:1), and the mixture was kept quietly for 24-48 hours. By evaporating of solvent crystals were precipitated. Crystals were separated by filter paper and recrystalliized from ethanol (95\%) water mixture. yield $0.9 \mathrm{~g}, 75 \%$. mp. $174^{\circ} \mathrm{C}$.

${ }^{1} \mathrm{H}$ NMR (300 MHz, DMSO-d6), $\delta$, m.h.: 1.72 (s, $\left.3 \mathrm{H}, \mathrm{CH}_{3}\right) ; 3.07$ (d, $1 \mathrm{H}, \mathrm{CH},{ }^{3} \mathrm{~J}_{\mathrm{H}-\mathrm{H}}$ $=11.7) ; 4.38\left(\mathrm{~d}, 1 \mathrm{H}, \mathrm{CH},{ }^{3} \mathrm{~J}_{\mathrm{H}-\mathrm{H}}=11.7\right) ; 7.00-$ 7.89 (m, 13H, 10Ar-H+3CH $\mathrm{CH}_{\text {thiophenyl) }}$; 9.86 (s, $1 \mathrm{H}, \mathrm{NH}) .{ }^{13} \mathrm{C}$ NMR spektr (DMSO-d6), $\delta$, m.h.: $26.38\left(\mathrm{CH}_{3}\right), 40.62(\mathrm{CH}), 55.87(\mathrm{CH})$, 86.25 (= $\left.\mathrm{C}_{\text {quat. }}\right), 99.73$ (O-C $\left.\mathrm{C}_{\text {quat. }}\right), 119.58(\mathrm{CN})$, $119.66\left(2 \mathrm{CH}_{\text {arom }}\right), 124.15\left(\mathrm{CH}_{\text {arom }}\right), 128.03$ $\left(\mathrm{CH}_{\text {arom }}\right), 128.47\left(\mathrm{CH}_{\text {thiophenyl }}\right), 128.60\left(\mathrm{CH}_{\text {arom }}\right)$, $128.87\left(2 \mathrm{CH}_{\text {arom }}\right), 129.05$ ( $\left.3 \mathrm{CH}_{\text {arom }}\right), 129.78$ $\left(\mathrm{CH}_{\text {thiophenyl }}\right), 130.63\left(\mathrm{CH}_{\text {thiophenyl }}\right), 135.99\left(\mathrm{C}_{\mathrm{ar}}\right)$, 138.87 ( $\left.\mathrm{C}_{\text {thioph }}\right), 140.12\left(\mathrm{C}_{\mathrm{ar}}\right), 166.77(\underline{\mathrm{CONH}})$, $167.63\left(\mathrm{O}-\mathrm{C}_{\text {quat. }}=\right)$.

Found, \%: $69.17 \quad \mathrm{C} ; 4.76 \quad \mathrm{H}$. $\mathrm{C}_{24} \mathrm{H}_{20} \mathrm{~N}_{2} \mathrm{O}_{3}$ S. Calculated, \%: $69.23 \mathrm{C} ; 4.81 \mathrm{H}$. 5-Cyano-2-hydroxy-2-methyl-N-phenyl-4(pyridin-4-yl)-6-(thiophen-2-yl)-3,4-dihydro-2H-pyran-3-carboxamide (11b):

Mixture 4-pyridinecarboxaldehyde or (2.9 mmol) and 2-thenoylacetonitrile (3 mmol) dissolved in $50 \mathrm{ml}$ of ethanol-water (4:1) was stirrered 5 minutes, reaction mixture was kept quietly for 36 hours. Then acetoacetanilide (3 mmol) was added to reaction mixture, stirred for 5 minutes and after 2-3 drop methylpiperazine was added and stirring was continued. Reaction progress was tracked by TLC (EtOAc/n-hexane, 2:1). Reaction mixture was kept quietly for 36 hours. Through evaporating of solvent it became possible to precipitate crystals. Crystals were separated by filter paper and recrystalliized from ethanol (95\%) water mixture. yield $0.97 \mathrm{~g}, \quad 79.51 \%$. $\mathrm{mp} .165^{\circ} \mathrm{C}$.

${ }^{1} \mathrm{H}$ NMR (300 MHz, DMSO-d6), $\delta$, m.h.: ${ }^{13} \mathrm{C}$ NMR spektr (DMSO- $d 6$ ), $\delta$, m.h.: $1.70\left(\mathrm{~s}, 3 \mathrm{H}, \mathrm{CH}_{3}\right) ; 3.05\left(\mathrm{~d}, 1 \mathrm{H}, \mathrm{CH},{ }^{3} \mathrm{~J}_{\mathrm{H}-\mathrm{H}}=\right.$ 11.4); 4.39 (d, $\left.1 \mathrm{H}, \mathrm{CH},{ }^{3} \mathrm{~J}_{\mathrm{H}-\mathrm{H}}=11.4\right)$; 7.03-8.56 $\left(\mathrm{m}, 12 \mathrm{H}, 9 \mathrm{Ar}-\mathrm{H}+3 \mathrm{CH}_{\text {thiophenyl }}\right) ; 9.88(\mathrm{~s}, 1 \mathrm{H}$, $\mathrm{NH}) .{ }^{13} \mathrm{C}$ NMR spektr (DMSO-d6), $\delta$, m.h.: $26.31\left(\mathrm{CH}_{3}\right), 40.21(\mathrm{CH}), 55.04(\mathrm{CH}), 84.45$ (= $\left.\mathrm{C}_{\text {quat. }}\right), 99.72$ (O-C $\left.\mathrm{C}_{\text {quat. }}\right), 119.71(\mathrm{CN}), 120.02$ (2CHarom $), 124.29\left(\mathrm{CH}_{\text {arom }}\right), 124.44\left(\mathrm{CH}_{\text {arom }}\right)$, $128.52\left(\mathrm{CH}_{\text {thiophenyl }}\right), 129.21\left(3 \mathrm{CH}_{\text {arom }}\right), 130.11$ $\left(\mathrm{CH}_{\text {thiophenyl }}\right), 130.97\left(\mathrm{CH}_{\text {thiophenyl }}\right), 135.67\left(\mathrm{C}_{\mathrm{ar}}\right)$, $138.68 \quad\left(\mathrm{C}_{\text {thioph }}\right), \quad 149.17 \quad\left(\mathrm{C}_{\mathrm{ar}}\right), \quad 150.38$ $\left(2 \mathrm{CH}_{\text {arom }}\right), \quad 166.68$ ( $\left.\underline{\mathrm{CONH}}\right), 167.23 \quad(\mathrm{O}-$ $\mathrm{C}_{\text {quat. }}=$ ).

Found, \%: $66.12 \quad \mathrm{C} ; 4.51 \mathrm{H}$. $\mathrm{C}_{23} \mathrm{H}_{19} \mathrm{~N}_{3} \mathrm{O}_{3} \mathrm{~S}$. Calculated, \%: $66.19 \mathrm{C} ; 4.56 \mathrm{H}$. 


\title{
REFERENCES
}

1. Aleksandra Pałasz. Synthesis of 3,4dihydro-2H-pyrans by hetero-Diels-Alder reactions of functionalized $\alpha, \beta$-unsaturated carbonyl compounds with N-vinyl-2oxazolidinone. Org. Biomol. Chem., 2005, vol.3, iss.17, pp. 3207-3212. doi: 10.1039/B504210K

2. Dhananjay B. Kendre, Raghunath B. Toche, Madhukar N. Jachak. Michael addition of dimedone with $\alpha, \beta$-unsaturated ketones: Synthesis of quinoline and chromene derivatives. Journal of Heterocyclic Compounds. 2008, vol. 45, iss. 3, pp. 667-671. doi: org/10.1002/jhet.5570450305

3. Qiongmei Zhang, Tong Fang, Xiaofeng Tong. Facile synthesis of highly functionalized six-membered heterocycles via $\mathrm{PPh}_{3}$-catalyzed [4+2] annulations of activated terminal alkynes and heterodienes: scope, mechanism, and application. Tetrahedron. 2010, vol. 66, iss. 40, pp. 8095-8100.

doi: org/10.1016/j.tet.2010.07.043

4. Huanzhen Ni, Weijun Yao, Abdul Waheed, Nisar Ullah, and Yixin Lu. Enantioselective [4+2]-Annulation of
Oxadienes and Allenones Catalyzed by an Amino Acid Derived Phosphine: Synthesis of Functionalized Dihydropyrans. Org. Lett., 2016, vol.18, iss. 9, pp.2138-2141. doi: 10.1021/acs.orglett.6b00760

5. Wen Liu, Jing Zhou, Changwu Zheng, Xingkuan Chen, Hua Xiao, Yingquan Yang, Yinlong Guo, Gang Zhao. Tandem cross-RauhuteCurrier/cyclization reactions of activated alkenes to give densely functionalized 3,4-dihydropyrans. Tetrahedron. 2011, vol. 67, iss.10, pp.1768-1773. doi: org/10.1016/j.tet.2011.01.036

6. Zhi-Peng Hu, Wei-Juan Wang, Xiao-Gang Yin, Xue-Jing Zhang, Ming Yan. Enantioselective synthesis of 2-amino-4H-pyrans via the organocatalytic cascade reaction of malononitrile and $\alpha$-substituted chalcones. Tetrahedron: Asymmetry. 2012, vol. 23, iss. 6-7, $\quad$ pp. 461-467. doi.org/10.1016/j.tetasy.2012.03.018

7. Maharramov A.M., Naghiyev F.N., Asgarova A.R., Rahimova A.G., Akhundova M.A., Mamedov I.G. Investigation of conversion various ilidenmalononitriles. Kimya Problemleri - Chemical Problems. 2018, no.1, pp. 69-72. (In Azerbaijan).

\section{TIOFEN OSASLI BӘZI KNOEVENAGEL ADDUKTLARININ ASETOASETANILIDLO REAKSIYASININ TODQIQI}

\author{
F.N. Nağıyev, A.M.Mohərramov, A.R. Osgərova, A.G. Rəhimova, \\ M.A. Axundova, I.G. Mamedov
}

Bakı Dövlat Universiteti

AZ 1148 Bakl, Z.Xalilov küç., 23; e-mail: farid.orgchemist@gmail.com

3-Fenil-2-(tiofen-2-karbonil)akrilonitril, 2-(tiofen-2-karbonil)-3-(p-tolil)akrilonitril va 3-(4-metoksifenil)-2-(tiofen-2-karbonil)akrilonitril, elaca do 3-piridinil-2-(tiofen-2-karbonil)akrilonitrilin asetoasetanilid ila Mixael birlaşma reaksiyasiyasından avazlanmiş heksanon va 3,4-dihidro-2H-piran töramalarinin alınması müayyan edirilmişdir. Sintez edilan birlaşmalain quruluşu NMR va RQA analiz metodlarının köməyila tasdiq olunmuşdur.

Açar sözlor: tiofen, akrilonitril, asetoasetanilid, piran, NMR 


\title{
ИССЛЕДОВАНИЕ РЕАКЦИИ ВЗАИМОДЕЙСТВИЯ НЕКОТОРЫХ АДДУКТОВ КНЕВЕНАГЕЛЯ НА ОСНОВЕ ТИОФЕНА С АЦЕТОАЦЕТАНИЛИДОМ
}

\author{
Ф.Н. Нагиев, А.М. Магеррамов, А.Р. Аскерова, А.Г. Рагимова, \\ М.А. Ахундова, И.Г. Мамедов \\ Бакинский государственный университет
}

AZ 1148 Баку, ул. 3.Халилова, 23; e-mail: farid.orgchemist@gmail.com

Путем реакиии присоединения по Михаэлю взаимодействием 3-фенил-2-(тиофен-2карбонил) акрилонитрила, 2-(тиофен-2-карбонил)-3-(n-толил)акрилонитрила и 3-(4метоксифенил)-2-(тиофен-2-карбонил)акрилонитрила, а также 3-пиридинил-2-(тиофен-2карбонил)акрилонитрила $c$ ачетоащетанилидом были получены соответствующие замещенные гексаноны $и$ 3,4-дигидро-2Н-пиран-производные. Структуры синтезированных соединений были подтверждены методами ЯМР и рентгеноструктурного анализа.

Ключевые слова: тиофен, акрилонитрил, ацетоацетанилид, пиран, ЯМР 